\title{
The Temporal Dynamics of Motor Memory across Wake and Sleep
}

\author{
Nicola Cellini ${ }^{1}$ and $\odot$ Elizabeth A. McDevitt ${ }^{2}$ \\ ${ }^{1}$ Department of General Psychology, University of Padova, 35131 Padova, Italy, and ${ }^{2}$ Department of Psychology, University of California Riverside, \\ Riverside, California 92521 \\ Review of Nettersheim et al.
}

People learn how to write, play piano, and ride a bicycle through repetitive practice. The acquisition of these procedural motor skills has been thought to occur in stages (Dayan and Cohen, 2011), with an initial fast learning phase characterized by rapid within-session improvement before reaching a performance plateau, followed by a period in which information is consolidated off-line. This off-line consolidation period is purported to stabilize fragile information, rendering it less susceptible to interference, and in some cases, to enhance the skill. Specifically, it has been proposed that stabilization of motor memory, which is defined as no change in performance from the level achieved during training, occurs during wakefulness, whereas enhancement, characterized by a performance gain, occurs during sleep (Walker, 2005).

This sleep-based enhancement theory emerged after several studies showed a gain in performance (up to $20 \%$ ) on motor memory tasks such as the sequential finger tapping task (FTT), when a period of sleep occurred between training and test sessions (Walker, 2005). However, the

Received June 24, 2015; revised July 29, 2015; accepted July 31, 2015.

We thank Sara C. Mednick for insightful comments on previous versions of this manuscript.

The authors declare no financial conflicts of interest.

Correspondence should be addressed to Nicola Cellini, Department of

General Psychology, University of Padova, Via Venezia 8, 35131 Padova, Italy. E-mail: cellini.nicola@gmail.com or nicola.cellini@unipd.it.

DOI:10.1523/JNEUROSCI.2429-15.2015

Copyright $\odot 2015$ the authors $\quad 0270-6474 / 15 / 3512085-03 \$ 15.00 / 0$ hypothesis has been challenged, and other studies have found that controlling for other variables such as fatigue and time since sleep may account for post-sleep motor skill enhancement (Pan and Rickard, 2015). For example, when short training blocks are used (i.e., $10 \mathrm{~s}$ rather than $30 \mathrm{~s}$ ), post-sleep improvement is no longer observed, possibly due to a reduction in the buildup of within-block performance fatigue (Rickard et al., 2008). Other results showed that if an FTT testing session occurred 5 or 30 min after a training session, there was an early boost in performance showing an increase of $\sim 20 \%$, the same magnitude of enhancement observed after a period of sleep (Hotermans et al., 2006). Together, these studies suggest that sleep helps stabilize but not enhance motor skills, and the time course of changes in a motor skill across wake and sleep is not as simple as originally thought.

In a recent study published in The Journal of Neuroscience, Nettersheim and colleagues (2015) aimed to further understand the dynamics of off-line consolidation, namely how motor skills change across time spent awake and how the effect of sleep may vary depending on time spent awake. The authors conducted a behavioral study modifying the classical FTT paradigm. Participants were required to type a sequence of five numbers (e.g., 4-13-2-4) as fast and accurately as possible with the fingers of their nondominant hand (see Walker, 2005). All subjects par- ticipated in both a wake and sleep condition. In one group, learning was followed by a $30 \mathrm{~min}$ period after which performance was tested or subjects went to sleep and were tested the next day. In the second group, participants spent $4 \mathrm{~h}$ awake after training, before being tested or going to sleep and being tested the next morning. Testing performance in the wake condition was considered a measure of pre-sleep performance, while testing performance after sleep indexed post-sleep learning. This design allowed Nettersheim et al. (2015) to investigate the early boost in performance (Hotermans et al., 2006) while avoiding an additional test session, which may act as a relearning or extra practice phase.

Results showed that motor skills are modified over the course of wake, independent of sleep. Participants who were tested $30 \mathrm{~min}$ after training showed an early boost in performance of $\sim 21 \%$. When testing was delayed until $4 \mathrm{~h}$ after training, no significant improvement was observed. Thus, these results indicate that motor skills improved on-line during training, with continued off-line improvement for at least $30 \mathrm{~min}$. However, between $30 \mathrm{~min}$ and $4 \mathrm{~h}$ after training, performance decayed to initial training levels and the early boost in performance was eliminated.

The two most important findings of the Nettersheim et al. (2015) study are (1) when sleep occurred 30 min after training, sleep prevented memory decay, 
stabilizing performance at the early boost level achieved during the first $30 \mathrm{~min}$ of post-training wake; and (2) when sleep occurred $4 \mathrm{~h}$ after training, performance was restored to the $30 \mathrm{~min}$ post-training early boost level, thereby eliminating the performance decay observed over 4 h of wake (Fig. 1). Thus, sleep did not introduce additional gains compared with the maximum performance level reached during wakefulness, but it preserved learning from decay and even restored learning that had already experienced decay.

Although the neural mechanisms underlying this sleep-related stabilization effect are unknown, a recent finding suggesting that sleep stabilizes memories by reducing dopaminergic signaling (Berry et al., 2015) may provide some insight. In this study, Drosophila underwent a conditioning paradigm, in which they learned that a specific odor was associated with an electrical shock. When tested in a T-maze, flies initially avoided the conditioned odor, but the memory quickly decayed during wakefulness. However, memory retention increased when flies were induced to sleep just after learning. At the same time, Berry et al. (2015) observed that during sleep the activity of MV1 dopaminergic neurons, which depends on behavioral states (i.e., increases during motor activity, decreases during resting periods), dramatically decreased. When these neurons were activated during wake by increased arousal, forgetting was enhanced in flies. Similarly, when these neurons were stimulated during sleep, there was increased forgetting. One possibility is that memory retention and forgetting depends on the level of arousal after learning (Mednick et al., 2011), which is closely tied to dopaminergic activity: in active states when dopaminergic activity is increased, memories are forgotten, whereas in periods of low motor activity (e.g., sleep and quiet rest), dopaminergic activity is decreased and the forgetting process is halted.

These findings offer a working model to explain the behavioral findings of Nettersheim et al. (2015) (Fig. 1). Through repetitive practice, humans can acquire a new motor skill, which reaches a performance plateau during training and receives an early boost after a short amount of time away from practice, which may be due to cellular consolidation processes such as early long-term potentiation (LTP) and synaptic morphogenesis (Hotermans et al., 2006). During subsequent active wakefulness, a decay process is ini-

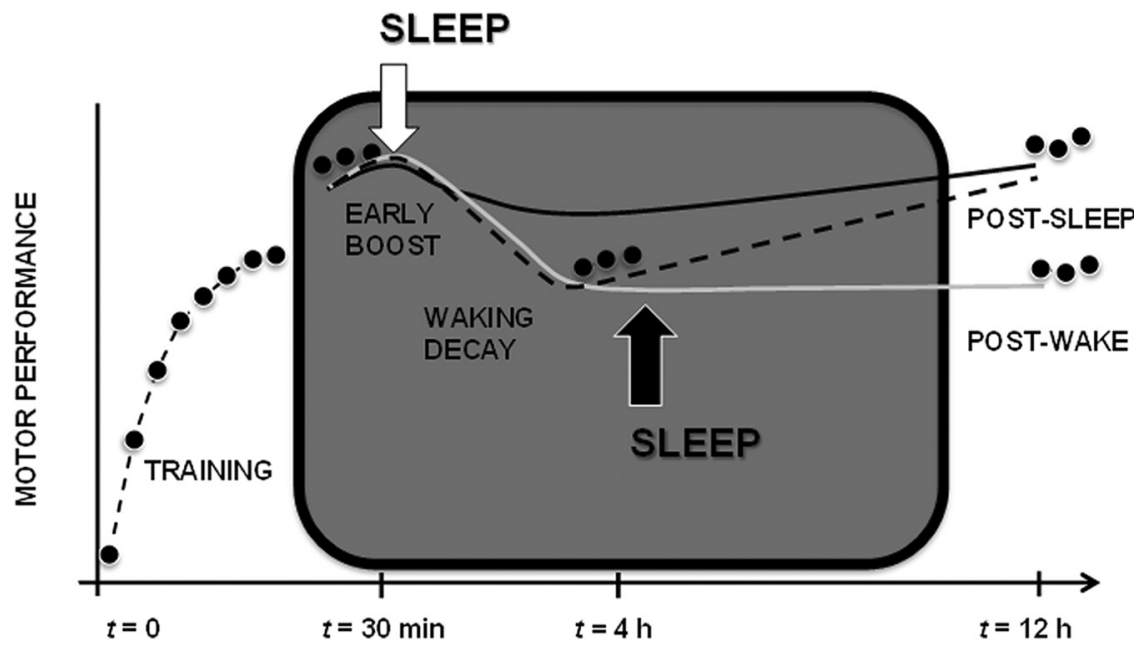

Figure 1. Nettersheim et al. (2015) showed that $\sim 30$ min after the end training, motor skills receive an early boost of $\sim 20 \%$ in performance. When followed by sleep (white arrow), information input is reduced and stabilization processes proceed, resulting in performance that is maintained at the enhanced early boost level (black line). Otherwise, performance subsequently decays over $4 \mathrm{~h}$ of wakefulness, possibly due to encoding of new information (i.e., interference). However, if sleep occurs at this time (black arrow), performance is restored to the early boost level (dashed line), suggesting that active, plasticity-related processes may work to restore motor memories. Without sleep, performance is no different than the performance plateau reached during initial training (gray line) (Walker, 2005).

tiated with concurrent encoding of new motor information (Mednick et al., 2011). These processes may be mediated by dopaminergic activity, which has been shown to modulate both LTP and longterm depression in cortical-striatum networks (Surmeier et al., 2007). However, if motor learning is followed by sleep, dopaminergic activity markedly decreases and the forgetting process is interrupted. This process, coupled with low plasticity (e.g., low acetylcholine) during non-REM sleep and low sensory and motor input may opportunistically allow the stabilization of the motor information (Mednick et al., 2011), which may be achieved through connectivity changes in the cortical-striatal system (Debas et al., 2014).

Nettersheim et al. (2015) also showed that sleep restored performance that had already experienced wake-induced decay, and indicated that it is "a matter of interpretation whether the reinstatement of an earlier performance level in the $4 \mathrm{~h}$ can be called 'stabilization' or whether it can be regarded as some form of enhancement" (p. 6701). Indeed, it is possible that sleep may also play an active role in consolidating disrupted memory traces. It has recently been shown that implicit perceptual learning damaged by retroactive interference before sleep can be rescued and consolidated during sleep, and that REM sleep, a sleep stage characterized by low information input coupled with high synaptic plasticity (e.g., high cholinergic tone), is critical to this process (McDevitt et al., 2015). Future studies testing the neurophysiological processes underlying the possible role of REM sleep in rescuing disrupted memories are warranted.

In summary, the elegant work of Nettersheim et al. (2015) provides behavioral evidence that sleep does not enhance motor skills (at least in the sense of true performance gains), but rather stabilizes them. Moreover, their study further supports the idea that sleep is able to rescue memories that have decayed over wake, restoring them to pre-decay levels. Notably, their behavioral results are consistent with a very recent quantitative metaanalysis involving 34 articles and $>1200$ participants (Pan and Rickard, 2015), suggesting that sleep does not produce a performance gain, but rather stabilizes motor memories. These results are also in line with independent human neuroimaging results (Debas et al., 2014), illustrating that consolidation of motor memories is associated with increased coherence in activity within the corticostriatal network when followed by a period of sleep, but not after an equivalent period of wake. Finally, the behavioral evidence presented by Nettersheim et al. (2015) also converges toward novel findings in flies showing that sleep inhibits specific dopamine neurons involved in forgetting mechanisms, which are very active during postlearning wakefulness, thereby promoting memory stabilization (Berry et al., 2015). Further work combining results from behavioral, neurophysiological, and im- 
aging studies will further advance our knowledge of how our brain learns and modifies motor skills.

\section{References}

Berry J, Cervantes-Sandoval I, Chakraborty M, Davis RL (2015) Sleep facilitates memory by blocking dopamine neuron-mediated forgetting. Cell 161:1656-1667. CrossRef Medline

Dayan E, Cohen LG (2011) Neuroplasticity subserving motor skill learning. Neuron 72:443454. CrossRef Medline

Debas K, Carrier J, Barakat M, Marrelec G, Bellec P, Hadj Tahar A, Karni A, Ungerleider LG, Benali H, Doyon J (2014) Off-line consolidation of motor sequence learning results in greater integration within a cortico-striatal functional network. Neuroimage 99:50-58. CrossRef Medline

Hotermans C, Peigneux P, Maertens de Noordhout A, Moonen G, Maquet P (2006) Early boost and slow consolidation in motor skill learning. Learning and Memory 13:580-583. CrossRef Medline

McDevitt EA, Duggan KA, Mednick SC (2015) REM sleep rescues learning from interference. Neurobiol Learn Mem 122:51-62. CrossRef Medline

Mednick SC, Cai DJ, Shuman T, Anagnostaras S, Wixted JT (2011) An opportunistic theory of cellular and systems consolidation. Trends Neurosci 34:504-514. CrossRef Medline

Nettersheim A, Hallschmid M, Born J, Diekelmann S (2015) The role of sleep in motor sequence consolidation: stabilization rather than enhancement. J Neurosci 35:6696-6702. CrossRef Medline

Pan SC, Rickard TC (2015) Sleep and motor learning: is there room for consolidation? Psychol Bull 141:812-834. CrossRef Medline

Rickard TC, Cai DJ, Rieth CA, Jones J, Ard MC (2008) Sleep does not enhance motor sequence learning. J Exp Psychol Learn Mem Cogn 34:834-842. CrossRef Medline

Surmeier DJ, Ding J, Day M, Wang Z, Shen W (2007) D1 and D2 dopamine-receptor modulation of striatal glutamatergic signaling in striatal medium spiny neurons. Trends Neurosci 30:228-235. CrossRef Medline

Walker MP (2005) A refined model of sleep and the time course of memory formation. Behav Brain Sci 28:51-64; discussion 64-104. Medline 